\title{
Rotura de válvula tricúspide con insuficiencia masiva secundaria a traumatismo torácico cerrado. Caso clínico
}

\author{
Roberto G onzález $L^{1}$, Ricardo Zalaquett $S^{1}$, \\ Gastón Chamorro $S^{1}$, G uillermo Lema $F^{2}$. \\ Massive tricuspid valve insufficiency \\ after blunt chest trauma. \\ Report of one case
}

Cardiac valve lesions after a blunt chest trauma are rare and less than $1 \%$ of cardiac lesions because of chest trauma affect the tricuspid valve. We report a 70 year-old female that suffered a severe chest trauma in a car accident. During the repair of the multiple skeletal lesions, the patient had a severe hemodynamic decompensation. A myocardial trauma with pericardial effusion and massive tricuspid insufficiency, due to anterior leaflet prolapse, was diagnosed on echocardiography. After discharge the patient remained in functional class II, with hepatomegaly, jugular ingurgitation and lower limb edema. A control echocardiogram, perfomed six months after the accident, showed dilatation of right heart chambers and massive tricuspid insufficiency. The patient was operated, and a tricuspid valve repair was performed suturing the ruptured papillary muscle to the ventricular wall and performing a triscuspid annuloplasty with a prosthetic ring. After 15 months of follow up, the patient remains asymptomatic (Rev Méd Chile 2008; 136: 1034-8).

(Key words: Heart failure; Shock, traumatic; Thoracic injuries; Tricuspid valve insufficiency)

Recibido el 10 de julio, 2007. Aceptado el 20 de diciembre, 2007.

${ }^{1}$ Departamento de Enfermedades Cardiovasculares. ${ }^{2}$ Anestesia Cardiovascular. Facultad de Medicina, Pontificia Universidad Católica de Chile. Santiago de Chile.

L

as lesiones valvulares cardiacas secundarias a Lraumatismos torácicos cerrados son en general poco frecuentes y, por su parte, las lesiones tricuspídeas son especialmente infrecuentes. Según las distintas series, menos de $1 \%$ de las lesiones cardiacas, tanto en traumatismos cerrados como abiertos, afectarían a la válvula tricúspide ${ }^{1,2}$.

Correspondencia a: Dr. Ricardo Zalaquett S. Hospital Clínico Pontificia Universidad Católica de Chile, Marcoleta 367, 6o piso, Santiago. Teléfonos: 6333030 - 6863231. Fax: 6390108. E mail: rzalaque@med.puc.cl
En la mayoría de los casos reportados, la insuficiencia tricuspídea traumática es secundaria a accidentes automovilísticos, aunque cualquier traumatismo torácico cerrado puede generar un daño estructural valvular tricuspídeo ${ }^{1-4}$. El mecanismo asociado sería el aumento súbito de la presión intracardiaca durante el sístole, provocado por la compresión del corazón entre el esternón y la columna vertebral al momento del accidente ${ }^{2,5}$.

Como frecuentemente se trata de pacientes politraumatizados, muchas veces su diagnóstico 
inicial no es fácil y las otras lesiones asociadas al traumatismo son evaluadas y tratadas en forma prioritaria, haciéndose tardíamente el diagnóstico de insuficiencia tricuspídea traumática ${ }^{1,6,7}$.

En el presente trabajo comunicamos un caso clínico de insuficiencia valvular tricuspídea masiva secundaria a rotura de músculo papilar por un traumatismo torácico cerrado, en el contexto de una paciente politraumatizada por un accidente automovilístico, que fue resuelto quirúrgicamente mediante una reparación valvular.

Caso clínico. Mujer de 70 años con antecedentes de hipertensión arterial e hipotiroidismo en tratamiento. En junio de 2005 sufre accidente automovilístico colisionando su automóvil contra un árbol. Presentó múltiples lesiones: 11 fracturas costales, hemoneumotórax bilateral, fractura esternal y luxofractura de cadera derecha que requirió tratamiento quirúrgico. Durante la intervención de sus lesiones esqueléticas la paciente presentó descompensación hemodinámica de difícil manejo, diagnosticándose posteriormente una contusión miocárdica con derrame pericárdico e insuficiencia tricuspídea masiva, por prolapso del velo anterior demostrada por ecocardiograma. Completó 30 días de hospitalización, 15 de los cuales fueron en Unidad de Cuidados Intensivos. Durante su evolución presentó, además, un tromboembolismo pulmonar, por lo que requirió el implante de un filtro en la vena cava inferior y se inició tratamiento anticoagulante, el que se extendió por 6 meses.

Recuperada de su politraumatismo y en rehabilitación por la lesión de su cadera derecha, se mantuvo en capacidad funcional II, presentando en el examen físico hepatomegalia, ingurgitación yugular y edema de extremidades inferiores.

El ecocardiograma transesofágico de control, efectuado a los 6 meses del accidente, demostró dilatación de cavidades derechas e insuficiencia tricuspídea masiva excéntrica por prolapso del velo anterior secundario a rotura de una cabeza del músculo papilar (Figura 1). Por este motivo se indicó cirugía, la que fue aceptada por la paciente. La coronariografía preoperatoria no demostró lesiones coronarias.

Procedimiento quirúrgico. Por esternotomía media vertical se expuso el corazón, que se encontraba adherido completamente al saco pericárdico. Las cavidades derechas y la arteria pulmonar estaban muy dilatadas. Luego de liberar las adherencias al pericardio, se conectó a circulación extracorpórea, con canulación en aorta ascendente y de ambas cavas por separado. Se administró solución de cardioplegia cristaloide anterógrada y una vez obtenido un paro electromécanico del corazón, se efectuó una auriculotomía derecha y se exploró la válvula tricúspide. Ésta tenía 3 velos

Figura 1. Ecocardiograma transesofágico pre reparación valvular. A. Prolapso velo tricuspídeo anterior por rotura de músculo papilar (flecha). B. Regurgitación tricuspídea masiva al color.

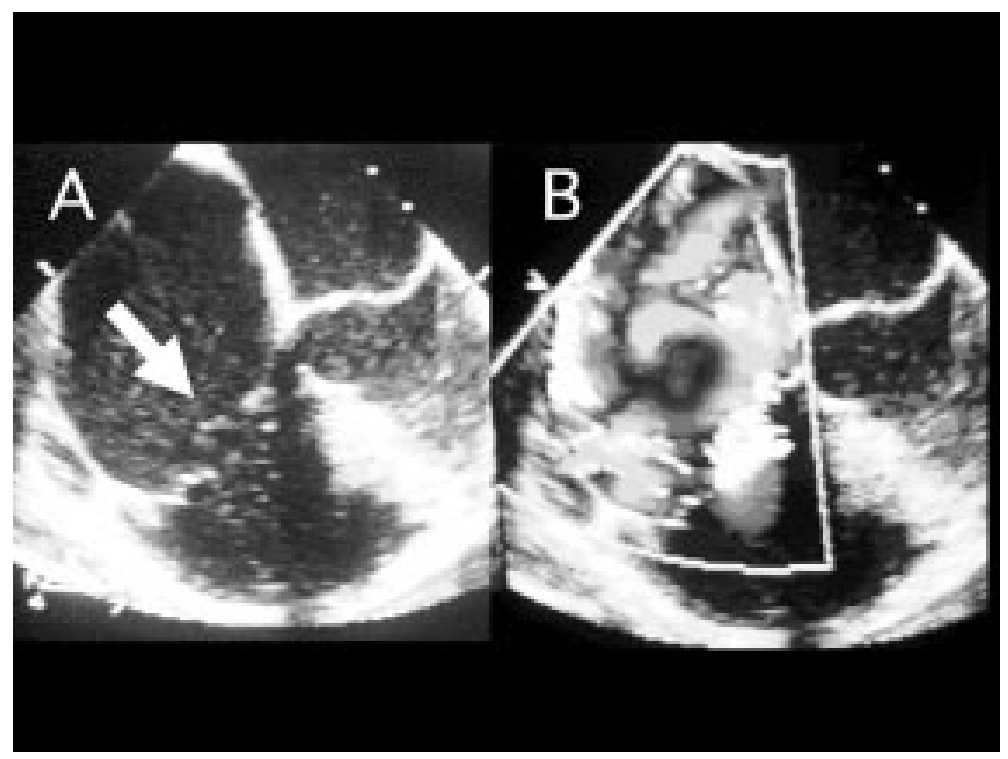




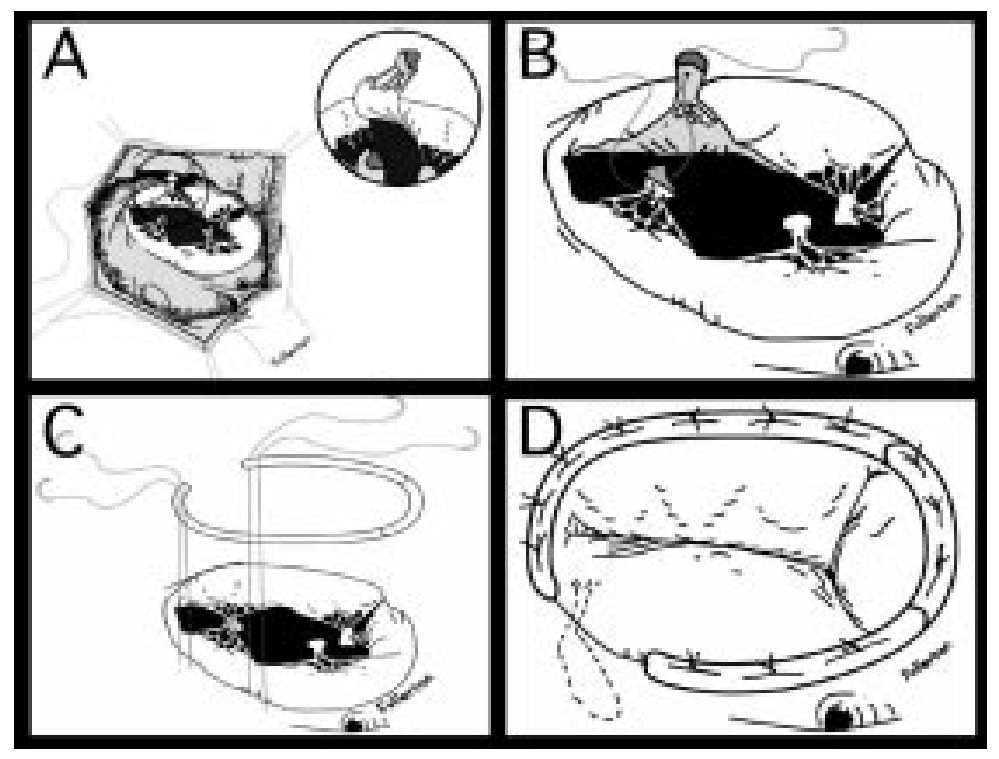

Figura 2. Esquemas intraoperatorios. A. Se esquematiza rotura de cabeza músculo papilar tricuspídeo. B. Se esquematiza la sutura de la cabeza del músculo papilar tricuspídeo rota. C. Se esquematiza la instalación del anillo protésico tricuspídeo que completa la reparación valvular. D. Aspecto final de la reparación valvular tricuspídea.

bien definidos y presentaba una dilatación importante del anillo, con una insuficiencia masiva al llenar el ventrículo derecho con solución salina isotónica. Se observó un prolapso de gran parte del velo anterior y de parte del septal por rotura de una pequeña cabeza del músculo papilar de aproximadamente $4 \mathrm{~mm}$, de la que se desprendían cuerdas tendíneas correspondientes a la zona del velo anterior inmediatamente adyacente a la comisura antero septal (Figura 2-A). Se suturó la cabeza del músculo papilar a una porción fibrosa del septum interventricular con puntos de Tycron 5-0, lo que se complementó con una anuloplastia tricuspídea con anillo de Carpentier-Edwards Clas$\operatorname{sic}^{\circledR}, \mathrm{n}^{\circ} 30$, para aumentar la coaptación de los velos y reducir los diámetros del anillo tricuspídeo, estabilizando la reparación valvular (Figuras 2-B, 2-C, 2-D). Se comprobó una buena continencia valvular al llenar el ventrículo derecho con solución salina isotónica. Además, se instaló un cable de marcapaso epicárdico definitivo, el que se tunelizó a un pequeño bolsillo subcutáneo en la pared abdominal, ante la eventualidad de un bloqueo aurículo-ventricular tardío.

El ecocardiograma transesofágico intraoperatorio demostró un buen aspecto de la reparación valvular, con insuficiencia tricuspídea residual mínima.
Evolución postoperatoria. La paciente evolucionó en forma muy satisfactoria y fue dada de alta al sexto día post operatorio. El ecocardiograma de superficie pre alta hospitalaria demostró un muy buen aspecto de la reparación valvular, sin insuficiencia tricuspídea residual. A 15 meses de seguimiento, la paciente se encuentra asintomática cardiovascular, en capacidad funcional I y sin insuficiencia tricuspídea en el ecocardiograma alejado.

\section{Discusión}

Las lesiones valvulares cardiacas secundarias a traumatismos cerrados son infrecuentes, siendo las más frecuentes las que involucran a la válvula aórtica, seguidas por las de la válvula mitral y en tercer lugar las de la tricúspide. A pesar de ser infrecuentes, deben siempre sospecharse, especialmente en el contexto de traumatismos de alta energía en pacientes politraumatizados ${ }^{1,5-7}$.

Las manifestaciones clínicas de la insuficiencia tricuspídea traumática pueden ser variadas y presentarse precozmente o mucho tiempo después del traumatismo. En general, corresponden a síntomas y signos de falla cardiaca derecha, que en muchos casos son bien tolerados, dependien- 
do de la cuantía de la insuficiencia. Clínicamente, la presencia de manifestaciones de falla cardiaca derecha en el contexto de un traumatismo torácico obliga a buscar las posibles causas de ésta, siendo el ecocardiograma fundamental en esta evaluación ${ }^{8-10}$.

El ecocardiograma juega un rol primordial en el estudio y caracterización de la insuficiencia tricuspídea, así como en la evaluación de otros daños estructurales cardiacos que se pueden asociar y en la estimación de la función ventricular derecha. Sin dudas, es el método diagnóstico de elección ${ }^{4,11-15}$.

Secundariamente, la insuficiencia tricuspídea lleva a una dilatación de las cavidades derechas, la que generalmente regresa después de corregida la insuficiencia tricuspídea, si bien muchos de los pacientes son sometidos a tratamiento quirúrgico varios años después del traumatismo, hasta 20 años según lo reportado en algunas comunicaciones $2,5,8,9$.

El momento de la cirugía en la insuficiencia tricuspídea secundaria a traumatismo torácico cerrado no está establecido y debe decidirse en forma individual, dependiendo de las manifestaciones clínicas y de los hallazgos ecocardiográficos de cada caso.

En una revisión de la literatura, Maisano et $\mathrm{al}^{2}$, en un total de 74 pacientes, describen que las lesiones tricuspídeas encontradas al momento de la cirugía fueron: rotura de cuerdas tendíneas en $55 \%$ de los casos, rotura del músculo papilar en $27 \%$ y rotura del velo en $15 \%$ de los casos. En todos, la rotura de cuerdas tendíneas del velo anterior fue la lesión más frecuente, presentándose en $42 \%$ de los pacientes.

Las alternativas quirúrgicas dependen de la anatomía patológica de la lesión y deben ser evaluadas según las características de cada pacien-

\section{REFERENCIAS}

1. Prêtre R, Chilcott M. Blunt trauma to the heart and great vessels. N Engl J Med 1997; 336: 626-32.

2. Maisano F, Lorusso R, Sandrem L, Torracca L, Coletti G, La CanNa G et al. Valve repair for traumatic tricuspid regurgitation. Eur J Cardiothorac Surg 1996; 10: 867-73. te. El reemplazo valvular con prótesis mecánica 0 biológica debe ser considerado sólo si existe un daño estructural que no permita la reparación 2,16,17.

Se han descrito múltiples alternativas de reparación, según sea el caso. Las más utilizadas son el implante de cuerdas tendíneas "artificiales", la reinsención de músculos papilares con 0 sin interposición de cuerdas artificiales y la comisuroplastía parcial, todas ellas complementadas con anuloplastía tricuspídea con anillo protésico ${ }^{2,18,20}$. En nuestra paciente, se realizó un reimplante de la cabeza de un músculo papilar desprendido, suturándola a la pared ventricular subyacente, y una anuloplastía tricuspídea con anillo CarpentierEdwars Classic. Además, se implantó un cable de marcapaso epicárdico ante la eventual necesidad de éste en el contexto de una reparación tricuspídea, situación descrita en otros casos ${ }^{7,16}$.

Los resultados de la reparación valvular en insuficiencia tricuspídea traumática son óptimos a corto y mediano plazo. Sin embargo, no existe un seguimiento alejado documentado por tratarse de casos aislados o series pequeñas. Nuestra paciente lleva un seguimiento de 15 meses y se encuentra sin insuficiencia tricuspídea clínica ni ecocardiográfica.

En conclusión, podemos decir que la insuficiencia tricuspídea traumática es una complicación infrecuente de los traumatismos torácicos cerrados, que puede tener una presentación clínica variable y manifestarse incluso alejadamente, por lo que es fundamental la sospecha clínica en el contexto de un paciente politraumatizado y que el tratamiento quirúrgico de elección debiera ser la reparación valvular.

Agradecimientos

Al Dr. Demian Fullerton M., por la confección de las figuras quirúrgicas. 
6. Kulik A, Al-Saigh M, Yele J, Rubens F. Subacute tricuspid valve rupture after traumatic cardiac and pulmonary contusions. Ann Thorac Surg 2006; 81: 1111-2.

7. Janzing H, Rommerns P, Flameng W, Aerts R, Lauwers $P$, Broos P. Severe liver rupture and tricuspid valve rupture in a patient with multiple trauma. J Trauma 1995; 38: 828-9.

8. Pasic M, Von Segesser L, Carrel T, Jenni R, Turina M. Severe tricuspid regurgitation following blunt chest trauma: indication for emergency surgery. Eur J Cardiothorac Surg 1992; 6: 455-7.

9. Fontes R, Salerno H, Lanzieri S, Oliveira S, Fernandez J, ECKERT I, ET AL. Insuficiência tricúspide pós trauma associada a agenesia de músculo papilar anterior. Rev Bras Cardiovas 2000; 15: 251-4.

10. Vazquez J, Diaz G, Flores O, Archundia A, Ugalde J. Presentación tardía de insuficiencia tricuspídea secundaria a trauma cardiaco contuso. An Med (Mex) 2006; 51: 80-3.

11. Banning A, Durrani A, Pilai R. Rupture of the atrial septum and tricuspid valve after blunt chest trauma. Ann Thorac Surg 1997; 64: 240-2.

12. Trotter T, KnotT-Craig C, Ward K. Blunt injury rupture of tricuspid valve and right coronary artery. Ann Thoracic Surg 1998; 66: 1814-6.

13. Reiss J, Razzouk J, Kiev J, Bansal R, Bailey L. Concomitant traumatic coronary artery and tricuspid valve injury: A heterogeneous presentation. J Trauma 2001; 50: 942-4.

14. Zakynthinos E, Vassilakopoulos T, Routsi C, Roussos C, ZAKYNTHINOS S. Early -and late- onset atrioventricular valve rupture after blunt chest trauma: The usefulness of transesophageal echocardiography. J Trauma 2002; 52: 990-6.

15. Chiu W, Shindeler D, Scholz P, Boyarsky A. Traumatic tricuspid regurgitation with cyanosis: Diagnosis by transesophageal echocardiography. Ann Thorac Surg 1996; 61: 992-3.

16. Veergandham R, Backer C, Mayroudis C, Wilson A. Traumatic left ventricular aneurysm and tricuspid insufficiency in a child. Ann Thorac Surg 1998; 66: 247-8.

17. Bertrand S, Laquay N, Rassi EI, Vouhé P. Tricuspid insufficiency after blunt chest trauma in a nine-yearold child. Eur J Cardiothorac Surg 1999; 16: 587-9.

18. BoLton J. Traumatic tricuspid Valve Injury: Leaflet resuspension repair. Ann Thorac Surg 1996; 61:721722.

19. VAN SON J, StARR A. Repair of traumatic valve insufficiency by trabecular muscle elevation. Ann Thoracic Surg 1995; 58: 740-2.

20. Noera G, Sanguinetti M, Pensa P, Biagi B, Cremonesi A, LODI R ET AL. Tricuspid valve incompetence caused by nonpenetrating thoracic trauma. Ann Thorac Surg 1991; 51: 320-2. 